\title{
Publisher Correction: Annual transcriptome dynamics in natural environments reveals plant seasonal adaptation
}

Atsushi J. Nagano (D), Tetsuhiro Kawagoe, Jiro Sugisaka, Mie N. Honjo, Koji Iwayama and Hiroshi Kudoh (D)

Correction to: Nature Plants https://doi.org/10.1038/s41477-018-0338-z, published online 9 January 2019.

In Fig. $3 \mathrm{~b}$ of the version of this Article originally published, a number of arrows indicating repression of downstream processes were mistakenly formatted as arrows indicating activation of downstream processes. The corrected figure is shown below. This has now been amended in all versions of the Article.
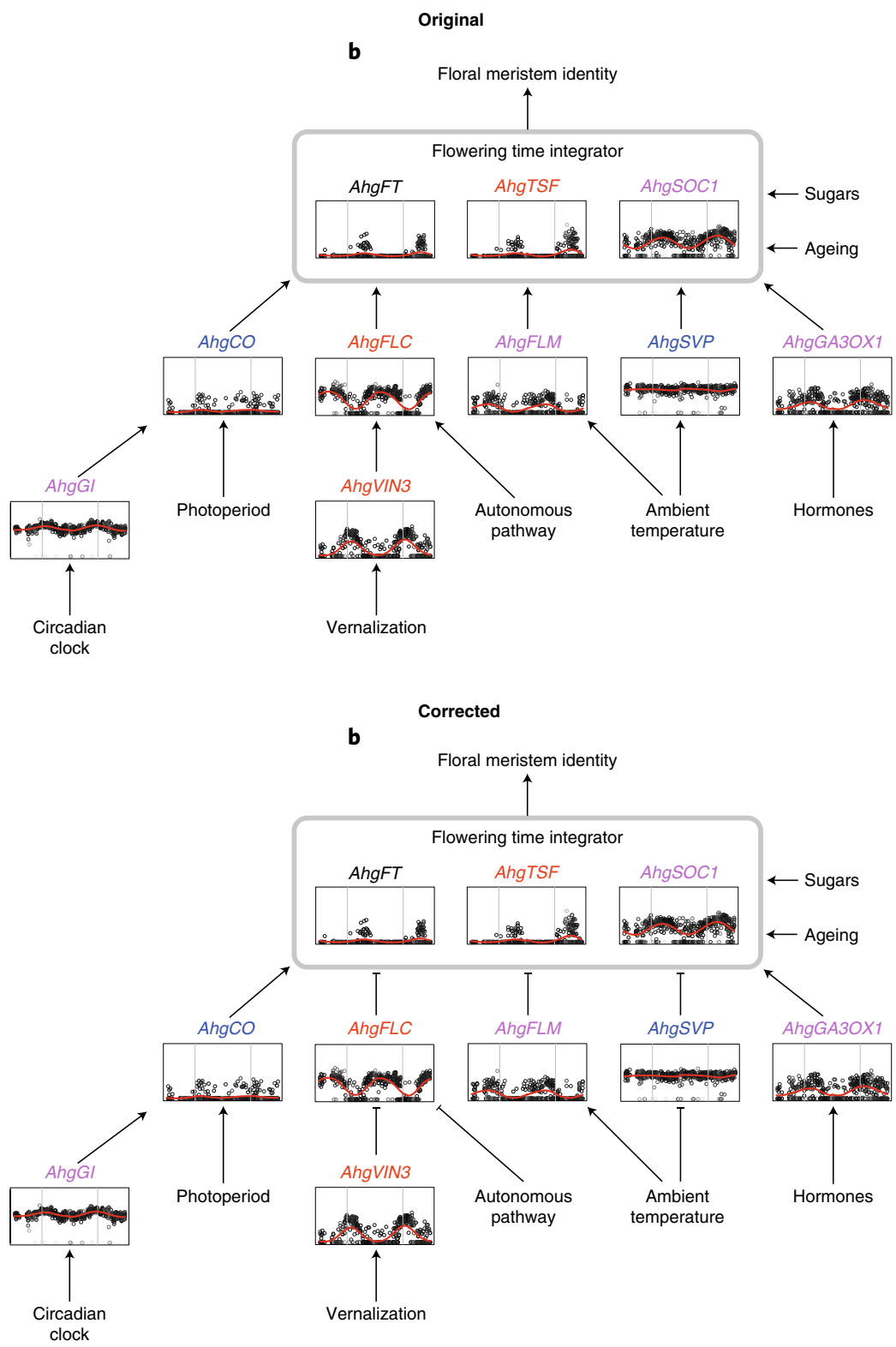

Fig. 3 | Original and Corrected 